JRRD

\title{
Human distal sciatic nerve fascicular anatomy: Implications for ankle control using nerve-cuff electrodes
}

\author{
Kenneth J. Gustafson, PhD; ${ }^{1-2 *}$ Yanina Grinberg, MS; ${ }^{1}$ Sheeba Joseph, BS; ${ }^{3}$ Ronald J. Triolo, PhD ${ }^{1-2,4}$ \\ ${ }^{1}$ Department of Biomedical Engineering, Case Western Reserve University, Cleveland, $\mathrm{OH} ;{ }^{2}$ Louis Stokes Cleveland \\ Department of Veterans Affairs Medical Center, Cleveland, $\mathrm{OH} ;{ }^{3}$ Case Western Reserve University School of Medicine, \\ Cleveland, $\mathrm{OH} ;{ }^{4}$ Department of Orthopedics, Case Western Reserve University, Cleveland, $\mathrm{OH}$
}

\begin{abstract}
The design of neural prostheses to restore standing balance, prevent foot drop, or provide active propulsion during ambulation requires detailed knowledge of the distal sciatic nerve anatomy. Three complete sciatic nerves and branches were dissected from the piriformis to each muscle entry point to characterize the branching patterns and diameters. Fascicle maps were created from serial sections of each distal terminus below the knee through the anastomosis of the tibial and common fibular nerves above the knee. Similar branching patterns and fascicle maps were observed across specimens. Fascicles innervating primary plantar flexors, dorsiflexors, invertors, and evertors were distinctly separate and functionally organized in the proximal tibial, common fibular, and distal sciatic nerves; however, fascicles from individual muscles were not apparent at these levels. The fascicular organization is conducive to selective stimulation for isolated and/or balanced dorsiflexion, plantar flexion, eversion, and inversion through a single multicontact nerve-cuff electrode. These neuroanatomical data are being used to design nerve-cuff electrodes for selective control of ankle movement and improve current lower-limb neural prostheses.
\end{abstract}

Key words: anatomy, ankle, common fibular, cuff, electrode, nerve, prosthesis, rehabilitation, sciatic, tibial.

\section{INTRODUCTION}

\section{Background}

Muscles innervated by the lower sciatic nerve control plantar flexion and dorsiflexion as well as inversion and eversion of the talocrural (ankle) joint and are therefore critical for standing balance and walking functions. The sciatic nerve originates in the lumbar and sacral spinal cord and supplies motor and sensory innervation to the lower limb. It has two major terminal branches, the tibial nerve and common fibular nerve. The common fibular (common peroneal) branches into the deep and superficial fibular (SF) nerves and is commonly targeted in neural prostheses used to correct foot drop [1-5]. The deep fibular (DF) branch innervates the tibialis anterior muscle, which dorsiflexes and inverts the foot. The DF nerve also innervates the extensor hallucis longus and extensor digitorum longus muscles, which primarily extend the toes and dorsiflex the foot, predominantly in non-weightbearing positions. Muscles innervated by the SF nerve (fibularis/peroneus longus and fibularis/peroneus brevis) evert and weakly plantarflex the foot and can counter the actions of tibialis anterior. The lateral sural cutaneous (LSC) and sural communicating branch nerves also originate

\footnotetext{
Abbreviations: $1 \mathrm{~L}=$ left side of cadaver $1,2 \mathrm{~L}=$ left side of cadaver $2,2 \mathrm{R}=$ right side of cadaver $2, \mathrm{DF}=$ deep fibular, $\mathrm{LSC}=$ lateral sural cutaneous, $\mathrm{MSC}=$ medial sural cutaneous, $\mathrm{NIH}=$ National Institutes of Health, SF = superficial fibular.

*Address all correspondence to Kenneth J. Gustafson, PhD; Case Western Reserve University Department of Biomedical Engineering, Neural Engineering Center, Wickenden Building, Rm 108, Cleveland, OH 44106-4912; 216368-8626; fax: 216-368-4872. Email: kjg@case.edu http://dx.doi.org/10.1682/JRRD.2010.10.0201
} 
from the common fibular nerve and have a sensory function that can elicit reflex activity. The tibial nerve innervates the soleus and gastrocnemius muscles, which plantar flex the foot, generate propulsive power for walking, provide a mechanism for the rocker actions of the foot and ankle, and retard uncontrolled tibial advancement. The gastrocnemius muscles also cross the knee joint and help avoid load bearing in hyperextension. Tibial nerve branches also innervate flexor digitorum longus and flexor hallucis longus, both of which flex the toes and plantar flex the foot, though mostly in a non-weightbearing position. The medial sural cutaneous (MSC) nerve, originating from the tibial nerve, has sensory properties that can elicit reflex activity.

\section{Neural Prostheses for Ankle Control}

Implantable neural prostheses for foot drop generally use nerve-cuff electrodes to activate the motor nerves and lift the foot, and some record sensory information to trigger stimulation [1-2,4,6-13]. However, most implantable neural prostheses provide only dorsiflexion to correct foot drop and do not provide active plantar flexion. Balanced dorsiflexion and active plantarflexion would improve the performance of neural prostheses for standing and walking after spinal cord injury and stroke. Detailed knowledge of the neuroanatomy and organization is required to design multicontact cuff electrodes able to selectively activate the muscles needed to achieve all of these functions and improve the functionality and cosmesis of lower-limb neural prostheses. Furthermore, accurate knowledge of target nerve morphology provides important design parameters for sizing and constructing cuff electrodes to avoid mechanical trauma and maximize stimulation efficiency [14-16].

The fascicular anatomy of two human sciatic nerves has been examined by McKinley [17] and Sunderland and Ray [18]. However, these studies do not provide adequate detail to allow nerve-cuff design. Techniques are available to selectively activate individual fascicles or groups within a nerve and to mathematically represent the neural behavior in response to stimulation [19-21]. Selective stimulation allows control of multiple distal muscles at a single proximal location. Modeling and simulation studies based on the detailed femoral fascicular anatomy [22] have been successfully employed to design and optimize implantable femoral nerve-cuff electrodes [16]. This model-driven approach to designing selective electrodes for the femoral nerve has been verified intraoperatively [23], and the resulting devices have been approved for human feasibility trials [24]. The first step in repeating this design and optimization procedure for the sciatic nerve is the specification of the fascicular structure of the branches that innervate ankle musculature.

The objectives of this study were to quantify the fascicular anatomy and morphology of the human lower sciatic nerve and to evaluate the potential of selective activation of ankle musculature with a multicontact nerve-cuff electrode. The fascicular structure of the lower sciatic nerve and the relationship of individual fascicles to distal nerve branches and terminal muscles will indicate the initial feasibility of selectively activating individual fascicles within the lower sciatic nerve using a single proximally located cuff electrode. This knowledge is critical for identifying the most desirable nerve-cuff electrode designs and placement sites.

\section{METHODS}

\section{Nerve Morphology}

Eight human sciatic nerves were examined in four formalin-fixed female cadavers (mean \pm standard deviation $92.5 \pm 5.7$; 87-102 years old). Distances from the greater trochanter to the knee joint line and from the joint line to the lateral malleolus were measured bilaterally on each cadaver and were used as anatomical landmarks. The inferior gluteal nerve branch point was chosen as the proximal limitation for surgical dissection of the upper sciatic nerve. Three lower sciatic nerves were dissected and harvested for detailed analysis of fascicular organization and structure. Measurements of nerve lengths with respect to bony landmarks were made during harvesting with the nerve in situ to allow for preliminary surgical planning.

Sciatic nerve branches were traced and dissected at their insertion points. Because of their small size and variable location, the popliteus, plantaris, and fibularis tertius (absent in some people) nerves were not always identified. Nerve branches were isolated from proximal to distal and superficial to deep to minimize damage to the nerves. As a nerve muscle entry point was isolated, the muscle was detached from its tendons and reflected. This process was repeated until all sciatic nerve branches were exposed. Each main branch was carefully tagged with a suture to maintain orientation and identification during the dissection process.

The branching patterns of three sciatic nerves were examined and characterized by the order of branching 
both medial to lateral and proximal to distal. The branchfree lengths and circumferences of the sciatic nerves and each branch were measured during extraction. Branchfree lengths were later confirmed using images of harvested nerves that were scaled with Adobe Photoshop CS (Adobe Systems Inc; San Jose, California) and ImageJ (National Institutes of Health [NIH]; Bethesda, Maryland) software. Branch-free lengths were tabulated as the linear distances between branch points. Circumferences were determined by measuring the length of a suture passed around the nerve just distal to branching off the sciatic, common fibular, or tibial nerves.

\section{Fascicular Anatomy}

Post harvesting, each sciatic nerve was stored in 10percent buffered formalin solution for preservation. Three sciatic nerves (left side of cadaver 1 [1L], left side of cadaver 2 [2L], right side of cadaver 2 [2R]) and their branches were sectioned to trace the fascicles from distal to proximal through the nerve. Five-millimeter-long transverse sections of the nerve were cut. Each section was marked with histopathology tissue dyes to indicate its orientation for processing and mapping. Sections were then embedded in paraffin and $5 \mu \mathrm{m}$ thick cross-sections were mounted on slides and stained with hematoxylin and eosin.

Digital images of each cross-section were taken through an Olympus BH-2 microscope with an Olympus DP10 camera (Olympus; Tokyo, Japan) at 1.67 to $40 \times$ magnification. Using Adobe Photoshop CS, digital images were compiled into maps of the sciatic nerve with the images oriented proximal to distal down the page. Fascicular groups going to the tibialis anterior, gastrocnemius, and soleus muscles were identified and traced distal to proximal on the map. Fascicles of the LSC, sural communicating branch, and MSC nerves were also traced for completeness. Groups of fascicles going to the tibial, common fibular, DF, and SF nerves were later identified and traced. Tracing distances of these fascicular groups with respect to specified landmarks were tabulated and compared across three nerves and compared to results reported by McKinley [17].

The number of fascicles in each nerve cross-section was counted. Trends in fascicular plexus formation and fascicular splitting within branch-free lengths were examined. The number of fascicles in all distal branches was found and compared with the number of fascicles in the lower sciatic immediately proximal to the bifurcation.

Nerve cross-sections from three lower sciatic nerves were outlined on digital images using modified NIH- developed ImageJ software. The area, major, and minor axes of each outlined nerve cross-section were determined. Effective diameter $\left(D_{\text {eff }}\right)$ of each cross-section was calculated from the area assuming a circular geometry: $D_{\text {eff }}=2 \times(\text { Area } / p)^{0.5}$. Measurements of multiple nerve branches innervating the same muscle in one specimen were averaged.

\section{RESULTS}

\section{Branching Patterns and Morphology}

The branching pattern of the sciatic nerve was compared across three nerves (1L, 2L, 2R). An example of a full harvested sciatic nerve (2R) with its terminal branches is shown in Figure 1(a). The pattern was fairly consistent with the exception of the branch innervating the long head of the biceps femoris muscle, which differed for one of the samples. The typical branching pattern of the sciatic nerve observed in this study is shown in Figure 1(b). The branching point of sural cutaneous nerves was proximal to the joint line in all eight specimens. The MSC nerve branched off the tibial nerve at approximately the same location as the branches innervating the soleus and gastrocnemius muscles, while the LSC and sural communicating branch nerves branched off the common fibular nerve proximal to the bifurcation. Branch-free lengths of the sciatic nerve and distal branches are given in the Table. Branch-free lengths of the sciatic, tibial, and common fibular nerves from the bifurcation to the first branch were all greater than $2 \mathrm{~cm}$, which is sufficient to accommodate currently available nerve-cuff electrodes. For clarification, the term distal sciatic nerve used hereafter refers to the sciatic nerve just prior to terminal bifurcation into the tibial and common fibular nerves.

The distances from the greater trochanter to the joint space of the knee and from the joint space to the lateral malleolus were $38.7 \pm 4.4 \mathrm{~cm}$ (range: $31.4-45 \mathrm{~cm}, n=8$ ) and $36.1 \pm 1.8 \mathrm{~cm}$ (range: $34.4-38.7 \mathrm{~cm}, n=8$ ), respectively. The bifurcation of the sciatic into the tibial and common fibular nerves occurred $8.1 \pm 2.1 \mathrm{~cm}$ (range: $4.5-$ $10.8 \mathrm{~cm}, n=8$ ) proximal to the joint space of the knee.

Average cross-section dimensions across three specimens of the sciatic, tibial, common fibular nerves, and their terminal branches are also shown in the Table. Nerves tended to be more elliptical than circular, with the major axis being 1.7 to 3.3 times larger than the minor axis, on average. 

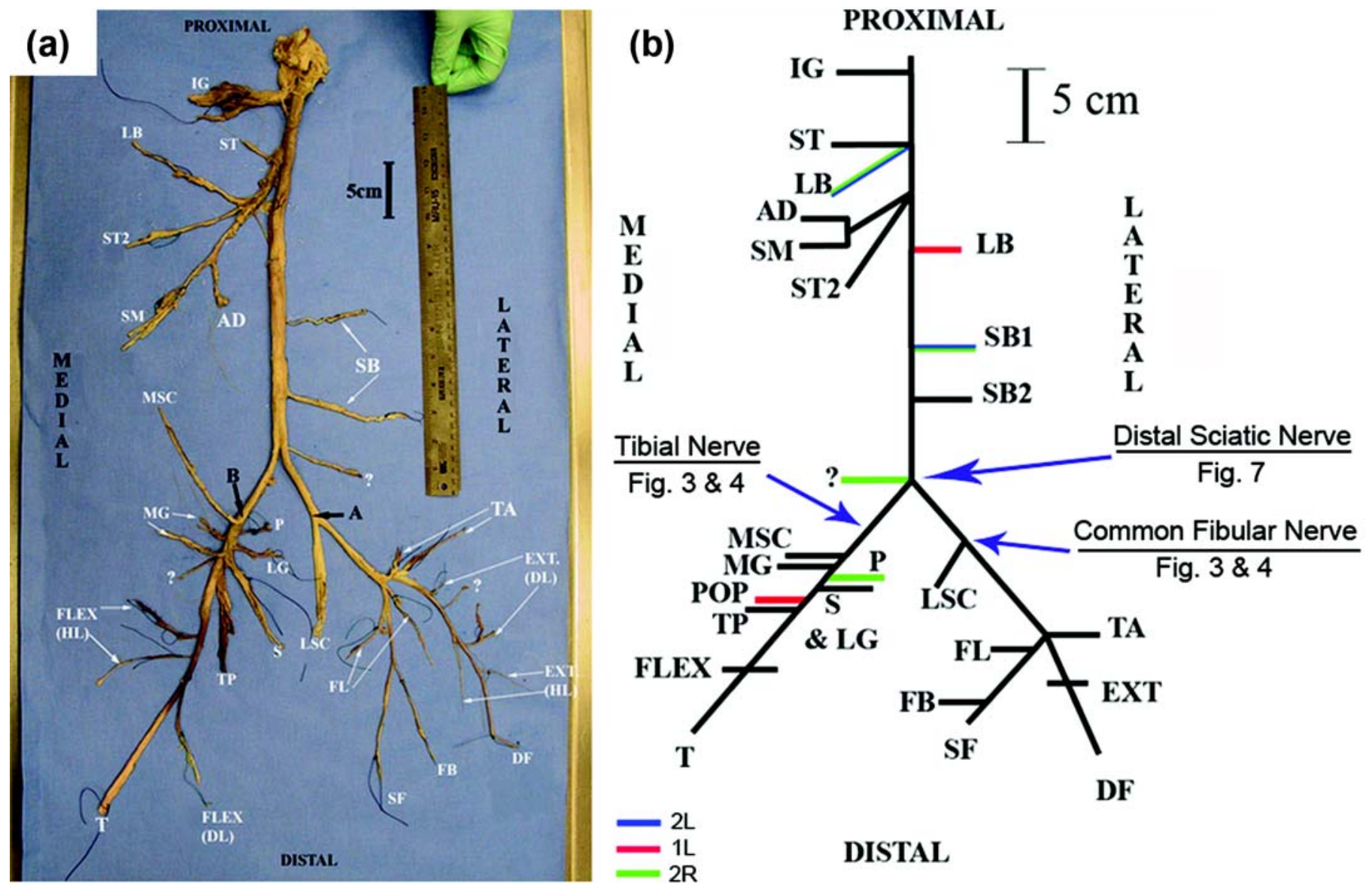

\section{Figure 1.}

Gross anatomy and stick-figure representation. Figure shows (a) harvested human sciatic nerve next to (b) average sciatic nerve seen in our study. Black branches are those that are common to all 3 specimens. Branches shown in red are only present in $1 \mathrm{~L}$, ones in green are seen in $2 \mathrm{R}$, and those in blue are present in $2 \mathrm{~L}$ only. ? = unidentified cutaneous branch, $1 \mathrm{~L}=$ left side of cadaver 1 , $2 \mathrm{~L}=$ left side of cadaver $2,2 \mathrm{R}=$ right side of cadaver $2, \mathrm{~A}=$ location of cross-sections presented in Figures 3-4, AD = adductor magnus, $B=$ location of cross-section presented in Figures 5-6, DF = deep fibular, DL = digitorum longus, EXT = extensors hallucis and digitorum longus, $F B=$ fibularis brevis, $F L=$ fibularis longus, FLEX = flexor hallucis and digitorum longus, $H L=$ hallucis longus, IG = inferior gluteal, LB = biceps femoris (long head), LG = lateral gastrocnemius, LSC/SCB ${ }^{*}=$ lateral sural cutaneous/sural communicating branch, MG = medial gastrocnemius, MSC = medial sural cutaneous, $\mathrm{P}=$ plantaris, $\mathrm{POP}=$ popliteus, $\mathrm{SB}=$ biceps femoris (short head), $\mathrm{SF}=$ superficial fibular, $\mathrm{SM}=$ semimembranosus, $\mathrm{S}=$ soleus, $\mathrm{ST}=$ semitendinosus, $\mathrm{T}=$ tibial nerve, $\mathrm{TA}=$ tibialis anterior, $\mathrm{TP}=$ tibialis posterior. Cross-sections shown in Figures 3, 4, and 7 were taken from respectively marked locations on (b). *After harvesting, this branch (LSC/SCB) was more accurately identified as SCB and the unidentified cutaneous branch (?) was determined to be LSC.

\section{Fascicular Anatomy}

Distal nerves were represented as individual fascicles or distinct fascicular groups and traced through sequential tissue sections to ultimately be outlined in proximal nerve sections; however, fascicular plexusing limited our ability to trace all individual fascicles proximally beyond the branching point of the tibial and common fibular nerves in all specimens. The most complete distal sciatic nerve was generated for specimen $2 \mathrm{R}$ and is shown in Figure 2. Also highlighted are the functionally significant nerve fascicles for the selective ankle-joint movement sought in this study.

\section{Fibular Nerve Fascicular Anatomy}

The common fibular nerve bifurcates into the DF and SF nerves, responsible for predominantly dorsiflexion with 
Table.

Sciatic nerve morphology.

\begin{tabular}{|c|c|c|c|c|c|c|}
\hline Nerve & $\begin{array}{l}\text { Branch-Free } \\
\text { Length }(\mathrm{cm})\end{array}$ & $\begin{array}{c}\text { Major Axis } \\
\text { (mm) }\end{array}$ & $\begin{array}{l}\text { Minor Axis } \\
\quad(\mathrm{mm})\end{array}$ & Major/Minor & Area $\left(\mathrm{mm}^{2}\right)$ & $\begin{array}{c}\text { Effective } \\
\text { Diameter } \\
(\mathrm{mm})^{*}\end{array}$ \\
\hline Upper Sciatic $^{\dagger}$ & $5.2 \pm 0.3$ & $14.7 \pm 1.4$ & $5.6 \pm 1.0$ & $2.7 \pm 0.7$ & $64.3 \pm 5.3$ & $9.0 \pm 0.4$ \\
\hline Semitendinosus & $9.5 \pm 1.6$ & 4.6 & 2.4 & 1.8 & 10.6 & 3.3 \\
\hline Biceps Femoris (long head) & $10.7 \pm 3.5$ & 3.5 & 1.4 & 2.5 & 3.8 & 2.2 \\
\hline Adductor Magnus & $6.9 \pm 3.7$ & 2.5 & 1.0 & 2.5 & 2.0 & 1.6 \\
\hline Biceps Femoris (short head) & $13.3 \pm 4.2$ & $4.8 \pm 2.2$ & $2.0 \pm 0.6$ & $2.4 \pm 0.4$ & $7.7 \pm 5.3$ & $3.0 \pm 1.1$ \\
\hline Distal Sciatic (proximal to bifurcation) & $5.1 \pm 1.5$ & $11.5 \pm 0.8$ & $6.4 \pm 1.5$ & $1.9 \pm 0.4$ & $58.2 \pm 17.5$ & $8.5 \pm 1.3$ \\
\hline Tibial & $7.4 \pm 1.0$ & $8.6 \pm 1.7$ & $5.2 \pm 0.3$ & $1.7 \pm 0.2$ & $35.5 \pm 8.6$ & $6.7 \pm 0.8$ \\
\hline Tibial $^{*}$ & - & $8.9 \pm 1.9$ & $5.4 \pm 0.4$ & $1.7 \pm 0.4$ & $37.5 \pm 6.9$ & $6.9 \pm 0.7$ \\
\hline Plantaris & 3.4 & - & - & - & - & - \\
\hline Soleus & $10.3 \pm 1.5$ & $3.3 \pm 0.8$ & $1.5 \pm 0.3$ & $2.2 \pm 0.0$ & $3.9 \pm 1.7$ & $2.2 \pm 0.5$ \\
\hline Lateral Gastrocnemius & $7.3 \pm 2.3$ & $2.8 \pm 0.4$ & $1.7 \pm 0.4$ & $1.7 \pm 0.5$ & $3.7 \pm 1.1$ & $2.1 \pm 0.3$ \\
\hline Popliteus & 4.6 & 2.8 & 0.7 & 4.1 & 1.5 & 1.4 \\
\hline Tibialis Posterior & $8.8 \pm 5.0$ & $4.4 \pm 2.4$ & $1.6 \pm 0.6$ & $2.6 \pm 0.6$ & $6.2 \pm 5.1$ & 1.8 \\
\hline Flexor Digitorum Longus & $8.0 \pm 2.3$ & $1.6 \pm 0.3$ & $0.8 \pm 0.4$ & $2.0 \pm 0.6$ & $1.1 \pm 0.6$ & 0.9 \\
\hline Flexor Hallucis Longus & $9.2 \pm 2.7$ & $2.1 \pm 0.0$ & $1.1 \pm 0.5$ & $2.1 \pm 1.0$ & $1.8 \pm 0.9$ & 1.8 \\
\hline Common Fibular & $5.0 \pm 2.2$ & $6.5 \pm 1.6$ & $3.3 \pm 0.5$ & $1.9 \pm 0.3$ & $16.9 \pm 5.9$ & $4.6 \pm 0.9$ \\
\hline Common Fibular $^{\dagger}$ & - & $6.9 \pm 0.8$ & $3.5 \pm 0.6$ & $2.0 \pm 0.1$ & $19.4 \pm 5.2$ & $4.9 \pm 0.7$ \\
\hline Extensor Digitorum Longus & $4.8 \pm 1.4$ & $1.7 \pm 0.3$ & $0.8 \pm 0.2$ & $2.1 \pm 0.2$ & $1.1 \pm 0.5$ & $1.2 \pm 0.3$ \\
\hline Extensor Hallucis Longus & $4.5 \pm 0.5$ & $2.2 \pm 1.7$ & $1.0 \pm 0.4$ & $2.0 \pm 0.9$ & $2.1 \pm 2.2$ & $1.5 \pm 0.8$ \\
\hline Proximal SF${ }^{\dagger}$ & $1.3 \pm 0.5$ & $3.7 \pm 1.9$ & $2.4 \pm 0.2$ & $1.5 \pm 0.7$ & $8.9 \pm 2.5$ & $3.3 \pm 0.5$ \\
\hline Fibularis Longus & $7.2 \pm 1.5$ & $2.7 \pm 0.8$ & $1.3 \pm 0.4$ & $2.1 \pm 0.3$ & $3.0 \pm 1.6$ & $1.9 \pm 0.6$ \\
\hline Fibularis Brevis & $7.1 \pm 1.8$ & $2.1 \pm 1.0$ & $1.3 \pm 0.7$ & $1.7 \pm 0.3$ & $2.5 \pm 2.5$ & $1.6 \pm 0.9$ \\
\hline \multicolumn{7}{|c|}{$\begin{array}{l}\text { Note: Unmarked measurements were taken immediately after branching point. Non-branch-free length data given in second through fifth rows of Upper Sciatic } \\
\text { portion of table are based on measurements from one specimen: } 1 \mathrm{~L} \text {. Data for plantaris and popliteus branches are based on one specimen each: } 2 \mathrm{R} \text { and } 1 \mathrm{~L} \text {, respec- } \\
\text { tively. All other data were gathered from three specimens. Data reported as mean } \pm \text { standard deviation. } \\
{ }^{*} \text { Derived assuming circular cross-sections. } \\
{ }^{\dagger} \text { Measurement taken in middle of branch-free length. } \\
\text { DF = deep fibular, dist = distal, LSC = lateral sural cutaneous, MSC = medial sural cutaneous, prox = proximal, SF = superficial fibular. }\end{array}$} \\
\hline
\end{tabular}

inversion and predominantly eversion, respectively. In all three specimens, the DF and SF remained distinguishable proximally through the common fibular nerve and through the sciatic bifurcation (joint space to bifurcation distance: $1 \mathrm{~L}=9.1 \mathrm{~cm}, 2 \mathrm{~L}=10.5 \mathrm{~cm}, 2 \mathrm{R}=10.8 \mathrm{~cm}$; distance proximal to bifurcation: $1 \mathrm{~L}=2.0 \mathrm{~cm}, 2 \mathrm{~L}=3.0 \mathrm{~cm}, 2 \mathrm{R}=3.5 \mathrm{~cm}$ ). 


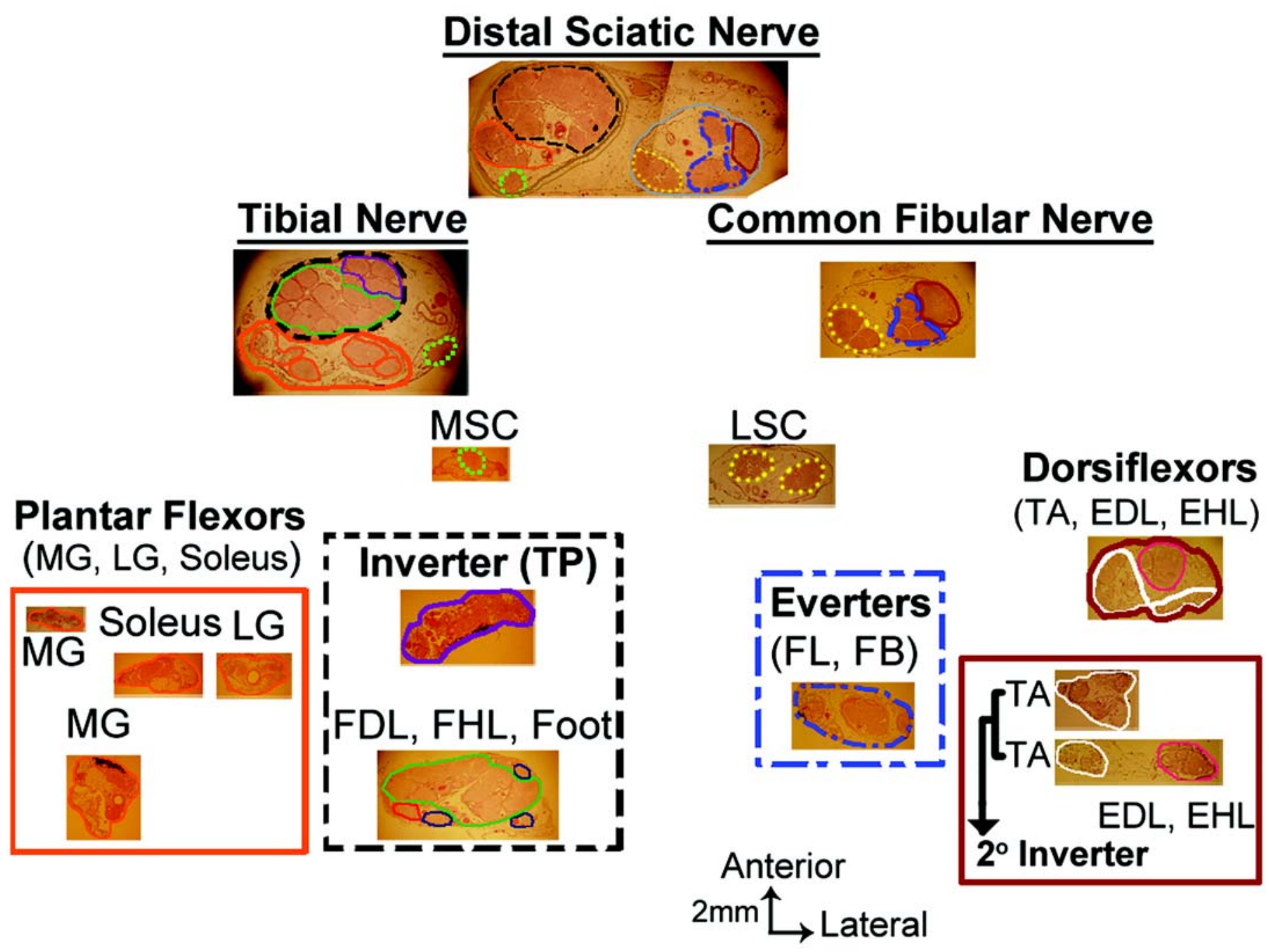

Plantar Flexors

\section{Common Fibular Nerve}

Dorsiflexors

Everters

Figure 2.

Fascicle map of compound sciatic nerve showing distal to proximal progression. Fascicular anatomy of lower sciatic nerve (2R) emphasizing functional divisions, with tibial branch divided into fascicles for plantar flexion (MG, LG, S), inversion (TP), and common peroneal branch divided into fascicles for inversion (TA), dorsiflexion (TA, EDL, EHL), and eversion (FL, FB). EDL = extensor digitorum longus, $\mathrm{EHL}=$ extensor hallucis longus, $\mathrm{FDL}=$ flexor digitorum longus, $\mathrm{FHL}=$ flexor hollucis longus, FL/FB = fibularis longus/ brevis, LSC = lateral sural cutaneous, MG/LG = medial/lateral gastrocnemius, MSC = medial sural cutaneous, TA = tibialis anterior, $\mathrm{TP}=$ tibialis posterior. Measurements are in millimeters.

The sural communicating and LSC nerve fascicles could be traced proximally through varying portions of the distal sciatic in all three specimens, being lost to interfascicular plexusing in specimen $1 \mathrm{~L}$ after $1.5 \mathrm{~cm}, 2 \mathrm{~L}$ after $2.0 \mathrm{~cm}$, and $2 \mathrm{R}$ after $2.5 \mathrm{~cm}$.

The fascicles to fibularis longus and fibularis brevis could not be distinguished separately after their anastomosis into the SF nerve because of interfascicular plexus- ing. The nerves to the extensor digitorum longus and extensor hallucis longus also could not be separated because of interfascicular plexusing. However, these two nerves as a group, along with the more distal DF nerve terminal branches in the foot, could be distinguished from the nerve to the tibialis anterior in two of the specimens (2L, 2R). This distinction could only be maintained for $5.5 \mathrm{~cm}(2 \mathrm{~L})$ and $6.5 \mathrm{~cm}(2 \mathrm{R})$ progressing proximally 
in the common fibular nerve. However, these portions of the nerve were just proximal to the joint space, $2 \mathrm{~L}$ by 0.5 $\mathrm{cm}$ and $2 \mathrm{R}$ by 1.5 to $2.0 \mathrm{~cm}$. The nerve to the tibialis anterior could not be traced separately proximal to its anastomosis with the remaining DF fascicles in one specimen (1L) because of interfascicular plexusing.

With regards to consistency across specimens, the general pattern observed found the DF and SF compound nerve fascicles located adjacent to each other and the sural cutaneous branch and the LSC located on the periphery. This pattern was observed in all three specimens, and was consistent with McKinley's study [17], though the latter had the DF and SF grouped together (Figure 3). Sunderland and Ray's tracing of the common fibular was detailed in the distal nerve, but the fascicles became intermixed more proximally, making functional grouping impossible [18], and hence was not shown for comparison. In addition to cross-specimen consistency, fascicular positioning was consistent along the length of the individual nerves themselves. This is shown in Figure 4 with specimen $1 \mathrm{~L}$.

\section{Tibial Nerve Fascicular Anatomy}

The tibial nerve initially gives off branches to innervate the gastrocnemius, the soleus, and the plantaris muscles-all of which function to plantar flex the talocrural joint. Thereafter, the tibial nerve continues distally to innervate deeper muscles and intrinsic muscles of the foot. The fascicles for the gastrocnemius and soleus muscles could not be separately identified proximal to their branch points from the tibial nerve in any of the samples. Given their similar function as weight-bearing plantar flexors, these fascicles were treated as a group for tracing purposes. This fascicular group for planter flexion was distinguishable proximally from the joint space past the sciatic bifurcation (joint space to bifurcation distance: $1 \mathrm{~L}=$ $9.1 \mathrm{~cm}, 2 \mathrm{~L}=10.5 \mathrm{~cm}, 2 \mathrm{R}=10.8 \mathrm{~cm}$; distance proximal to bifurcation: $1 \mathrm{~L}=6.0 \mathrm{~cm}, 2 \mathrm{~L}=5.0 \mathrm{~cm}, 2 \mathrm{R}=6.0 \mathrm{~cm}$ ).

The MSC nerve could be traced proximally and separately through the tibial nerve and into the sciatic nerve with certainty in two (2L, 2R) of the three samples. In $1 \mathrm{~L}$, the labeling was less certain. It was possible to trace the MSC proximally from the anastomosis of the tibial nerve and common fibular into the sciatic nerve for about $1 \mathrm{~cm}$, after which point interfascicular plexusing made separation difficult.

When comparing tibial nerve anatomy across specimens, consistency was evident between the group of fascicles innervating the gastrocnemius, soleus, and MSC
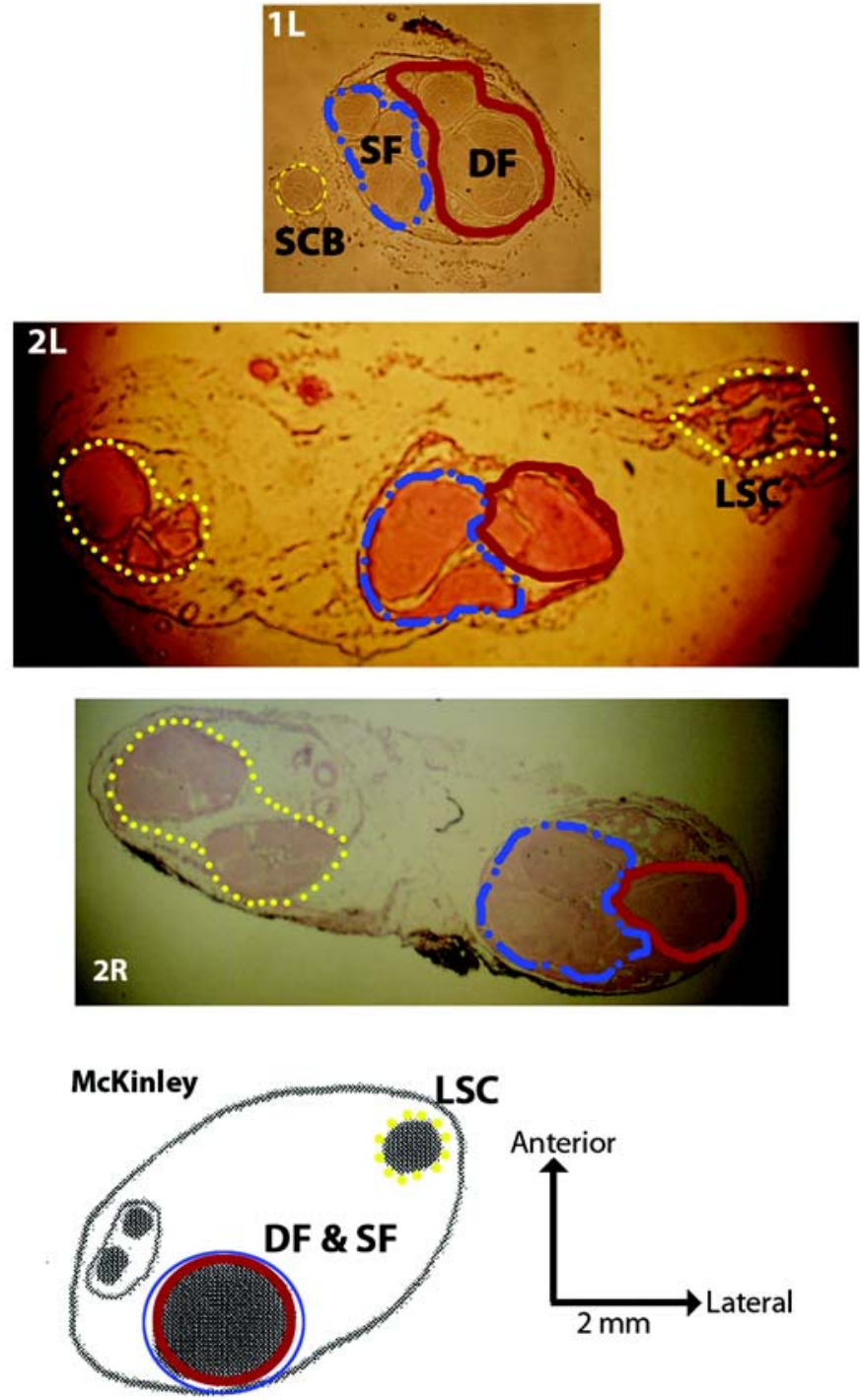

Figure 3.

Common fibular nerve cross-section comparison. Figure compares cross-sections of four human common fibular nerves (including McKinley JC. The intraneural plexus of fasciculi and fibers in the sciatic nerve. Arch Neurol Psychiatry. 1921; 6(4):377-99. Copyright (c) (1921) American Medical Association. All rights reserved.) proximal to joint space. Each crosssection is oriented with lateral side on right and anterior on top. Sural communicating branch (SCB) and lateral sural cutaneous (LSC) nerves are outlined in dotted yellow and are separable in all four nerves. Deep fibular (DF) is outlined in thick red and superficial fibular (SF) in dash-dotted blue. McKinley was not able to separate DF and SF at this level; therefore, both of these groups are circled with blue and red. 


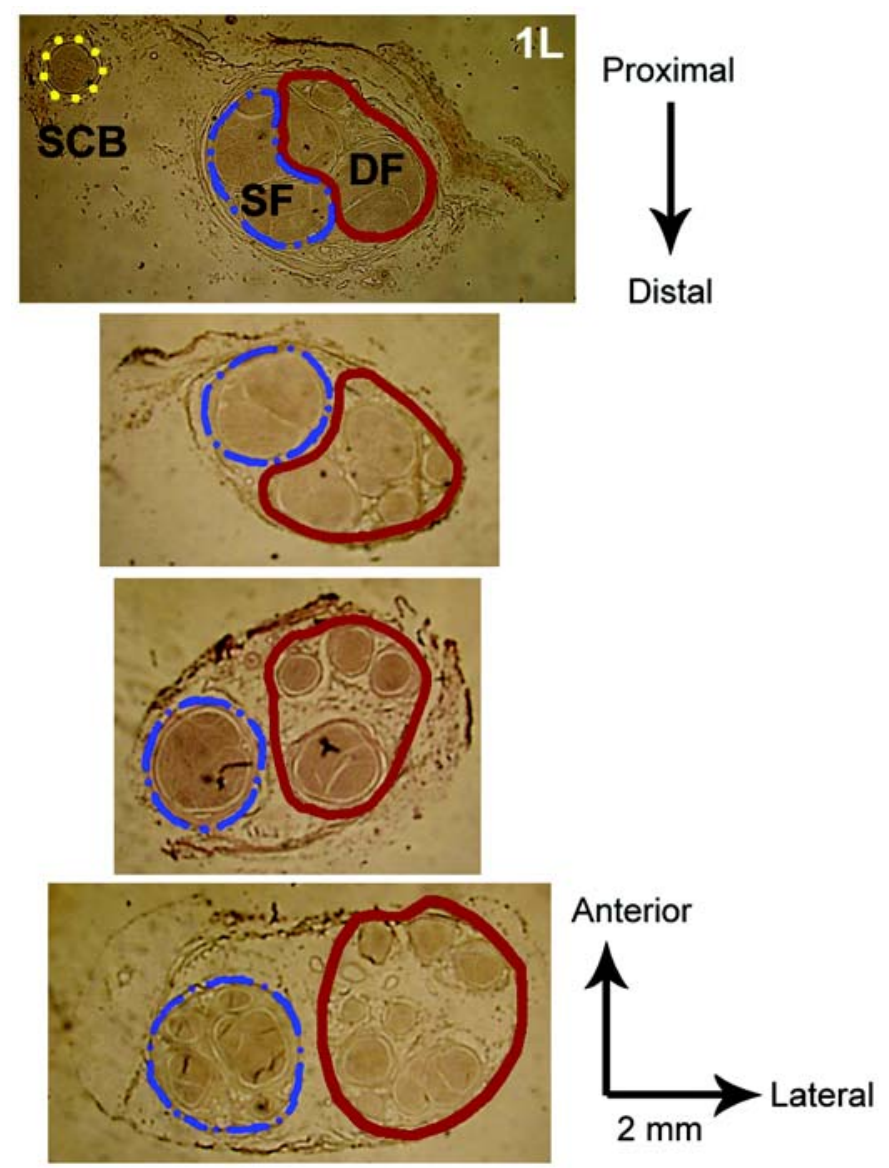

Figure 4.

Consistency in fascicular organization of common fibular nerve. Four cross-sections from one common fibular nerve (1L) taken distal to sciatic bifurcation and proximal to joint space of knee over distance of $6.5 \mathrm{~cm}$ are compared. Fascicles outlined in thick red are going to deep fibular (DF) and are crucial for foot dorsiflexion. Fascicles outlined in dash-dotted blue are going to superficial fibular (SF), and those outlined with a dotted yellow line are going to sural communicating branch (SCB). Nerve sections are oriented with lateral side to right and anterior side to top. Most proximal section is located at top of figure. $1 \mathrm{~L}=$ left side of cadaver 1.

located posterolaterally and the group of fascicles innervating the intrinsic foot muscles located more anteromedially. This is shown in Figure 5, which also includes a sample harvested by McKinley [17] showing similar fascicular organization. Sunderland and Ray's tracings did not maintain separate, functionally distinct groups in any sections proximal to the joint space [18] and are not shown for comparison. In addition to cross-specimen consistency, fascicu-

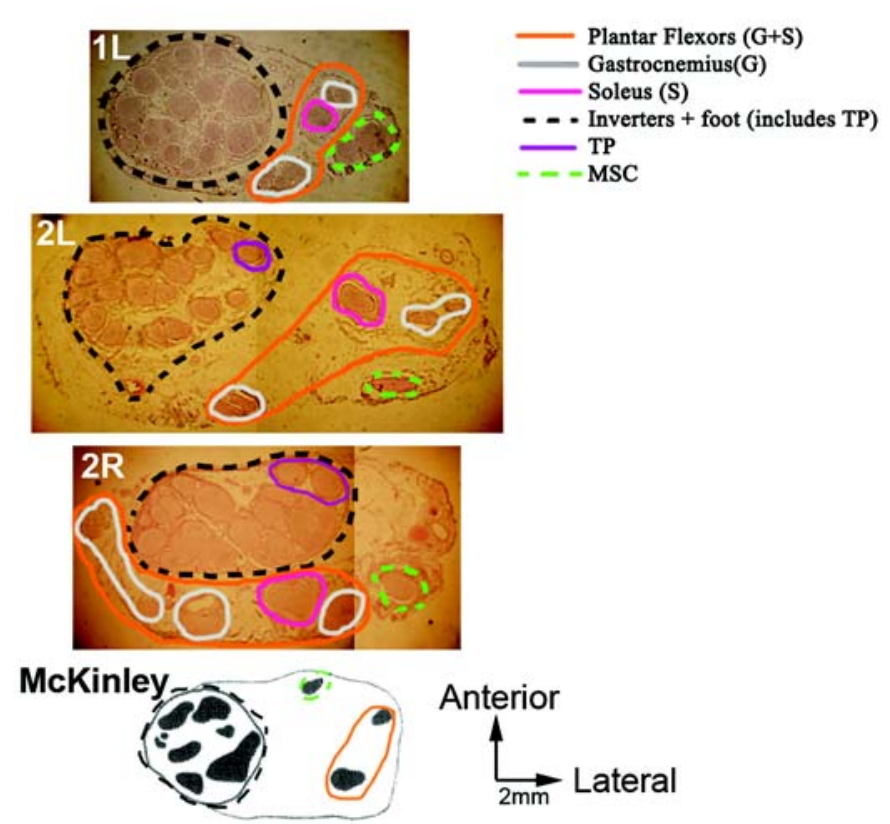

Figure 5.

Tibial nerve cross-section comparison. Comparison of crosssections of four human tibial nerves (including McKinley JC. The intraneural plexus of fasciculi and fibers in the sciatic nerve. Arch Neurol Psychiatry. 1921;6(4):377-99. Copyright $\odot$ (1921) American Medical Association. All rights reserved.) proximal to branching of medial sural cutaneous (MSC) nerve. Each cross-section is oriented with lateral side on right and anterior on top. Fascicles going to gastrocnemius, soleus, and MSC are circled in thick orange, while all other fascicles of tibial nerve are outlined in dashed black. MSC could be separated in all samples and is circled in dashed green. TP = tibialis posterior.

lar anatomy appeared consistent within the individual nerves themselves, as shown in Figure 6 with specimen 2L.

\section{Distal Sciatic Nerve Fascicular Anatomy}

Fascicles of the common fibular nerve were separable from those of the tibial nerve in the distal sciatic nerve. There was consistency across the specimens in the distal sciatic nerve within $2 \mathrm{~cm}$ proximal to the bifurcation, such that tibial nerve fascicles were located medially and common fibular nerve fascicles were located laterally. The DF and SF nerve fascicles could be identified in the distal sciatic nerve in all three samples. The fascicles innervating musculature for plantar flexion (gastrocnemius and soleus) were distinguishable in the distal sciatic nerve. Cutaneous nerve fascicles (MSC, 


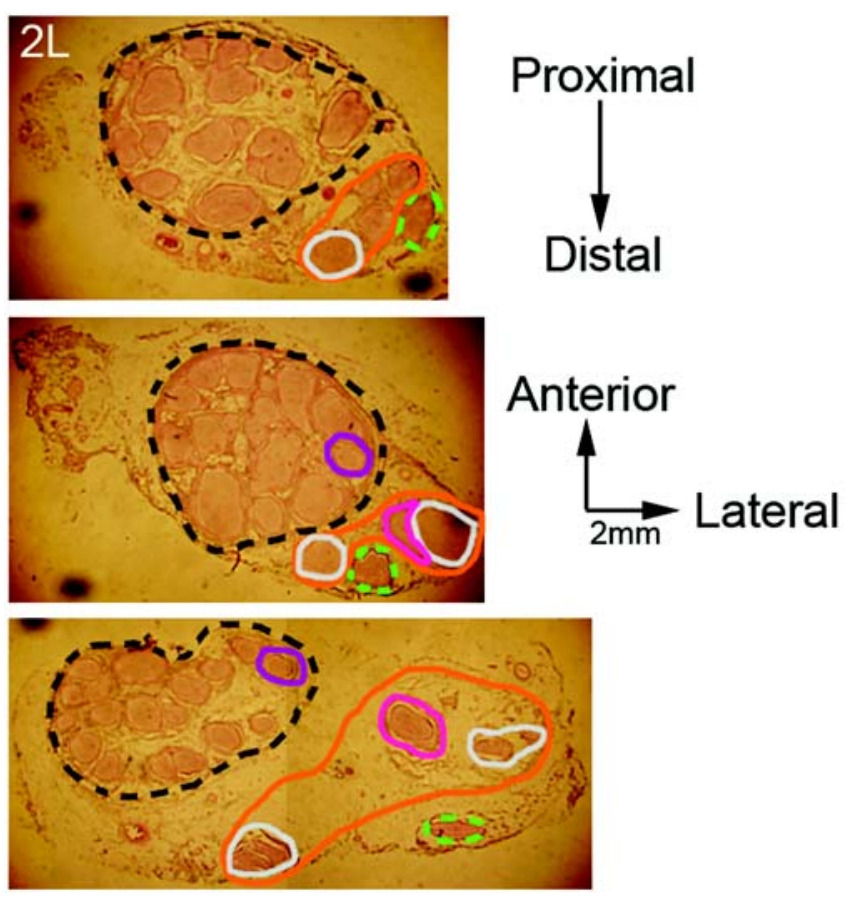

Figure 6.

Consistency in fascicular organization of tibial nerve. Three cross-sections from tibial nerve (2L) taken distal to sciatic bifurcation and proximal to joint space of knee over distance of $6.5 \mathrm{~cm}$ are compared. Fascicles going to gastrocnemius and soleus muscles as well as to medial sural cutaneous are outlined in thick orange. This fascicular group is consistently on posterior/lateral side of nerve. All other fascicles are outlined in dashed black. Nerve sections are oriented with lateral side to right and anterior side to top. Most proximal section is located at top of figure.

LSC, and sural communicating branch) were also identifiable in the sciatic nerve, with the exception of identifying the MSC and LSC in one sample (1L). Given the indistinct groupings of fascicles in Sunderland and Ray's cross-sections at the level of the tibial and common fibular nerves, distinct functional groups were not evident at the level of the distal sciatic [18].

\section{DISCUSSION}

This study quantified the fascicular anatomy and morphology of the human distal sciatic nerve and the common fibular and tibial nerves. These results from three specimens expand our current knowledge from two previous specimens [17-18]. The fascicular anatomy and morphology prove conducive to selective activation of ankle musculature and the generation of balanced dorsiflexion and plantarflexion with multicontact nerve-cuff electrodes. Selective activation would improve current neuroprosthetic systems for standing and walking after paralysis.

\section{Anatomy and Morphology}

The nerve branching patterns were consistent with general morphology [25] and the two previous studies, McKinley [17] and Sunderland and Ray [18], with minor variations. There is sufficient branch-free length of the distal sciatic proximal to the bifurcation for nerve-cuff placement (Table: $5.1 \pm 1.5 \mathrm{~cm}$; range 3.7-6.7 cm). McKinley reported this distance as $12 \mathrm{~cm}$ [17], and variability in the location of the sciatic bifurcation has been documented [26]. The branch-free lengths of the common fibular and tibial nerves were greater than $2 \mathrm{~cm}$, also making them sufficient for current nerve-cuff electrodes (Table). These three nerve locations are all above the knee, which is desirable to minimize the risk of mechanical damage and lead wire fatigue fracture.

Our measurements of nerve diameter and crosssectional areas are consistent with those reported by Bodily et al. [27]. However, dimensions of cross-sections in the nerve (sex unknown) examined by McKinley were consistently smaller than those found in our study [17]. We only examined female cadavers; nerve dimensions may be larger in men, who are more common candidates for neural prostheses for restoration of standing. The nerve dimensions may underestimate in vivo dimensions due to using formalin fixed cadavers and histological processing.

\section{Fascicular Anatomy and Mapping}

We were able to trace fascicles within the common fibular and tibial nerves; however, tracing all distal branches through the level of the distal sciatic was not possible in all specimens at the level of resolution (4-5 mm) used in this study. Higher resolution sectioning $(250 \mu \mathrm{m})$ improved tracing or identifying interfascicular plexusing in some nerve tissue sections. The observed fascicular anatomy was generally consistent with previous work [17-18] at the distal branches of the sciatic, below the joint space. McKinley did not identify subgroups proximal to the sciatic bifurcation [17], and Sunderland and Ray were unable to distinctly trace unique fascicles above the joint space because of interfascicular plexusing [18]. Although early studies reported difficulties tracing fascicular anatomy along nerves [18], more recent work [28] and work from our laboratory [22,29] has demonstrated 
that fascicular tracing is consistent in a number of peripheral nerves.

Both McKinley and Sunderland and Ray concluded that little functional grouping of motor fibers occurs other than that of the two main divisions, the common fibular and tibial nerves [17-18]. Our results showed that fascicles innervating subgroups of these main divisions could be identified as functional groups within the common fibular nerve and the tibial nerve (Figures 3 and 5). The DF and SF nerves were identifiable as individual fascicular groups within the common fibular nerve and within distal portions of the sciatic nerve (Figures 3 and 7), indicating feasibility to separately target muscles primarily for dorsiflexion from those primarily for eversion. O'Halloran et al. reported fascicles in the common fibular nerve being organized in a comparatively mixed pattern, with fascicles from both the DF and SF nerves grouped together [9]. Tracing distal branches of the tibial nerve to the lower sciatic was possible for functional groups of nerves such as the group of primary, weightbearing plantar flexors (gastrocnemius and soleus) and the group including an inverter and weak plantar flexor (tibialis posterior) and other weak plantar flexors (flexor digitorum longus and flexor hallucis longus) (Figures 5 and 7).

\section{Clinical Implications}

The anatomy and fascicular structure of the human distal sciatic, common fibular, and tibial nerves are conducive to selective stimulation with multicontact nervecuff electrodes. Separation of functionally organized groups of fascicles producing dorsiflexion, plantar flexion, inversion, and eversion suggests that these groupings can be selectively electrically stimulated. These data allow evaluation of interfacing locations such as the distal sciatic, proximal common fibular, and proximal tibial nerves. Nerve-cuff placement as far distally as possible ensures the best muscle activation selectivity; however, locations below the knee may have complications caused by lead wires crossing the joint [30]. Cuff placement proximal to the joint space may be more surgically accessible or stable. Modeling studies may be required to compare the efficacy of a single distal sciatic electrode versus two electrodes on the common fibular nerve and the tibial nerves.

In the proximal common fibular nerve, the DF, SF, and cutaneous branches (sural communicating branch and/or LSC branch) could be distinguished separately (Figure 3).
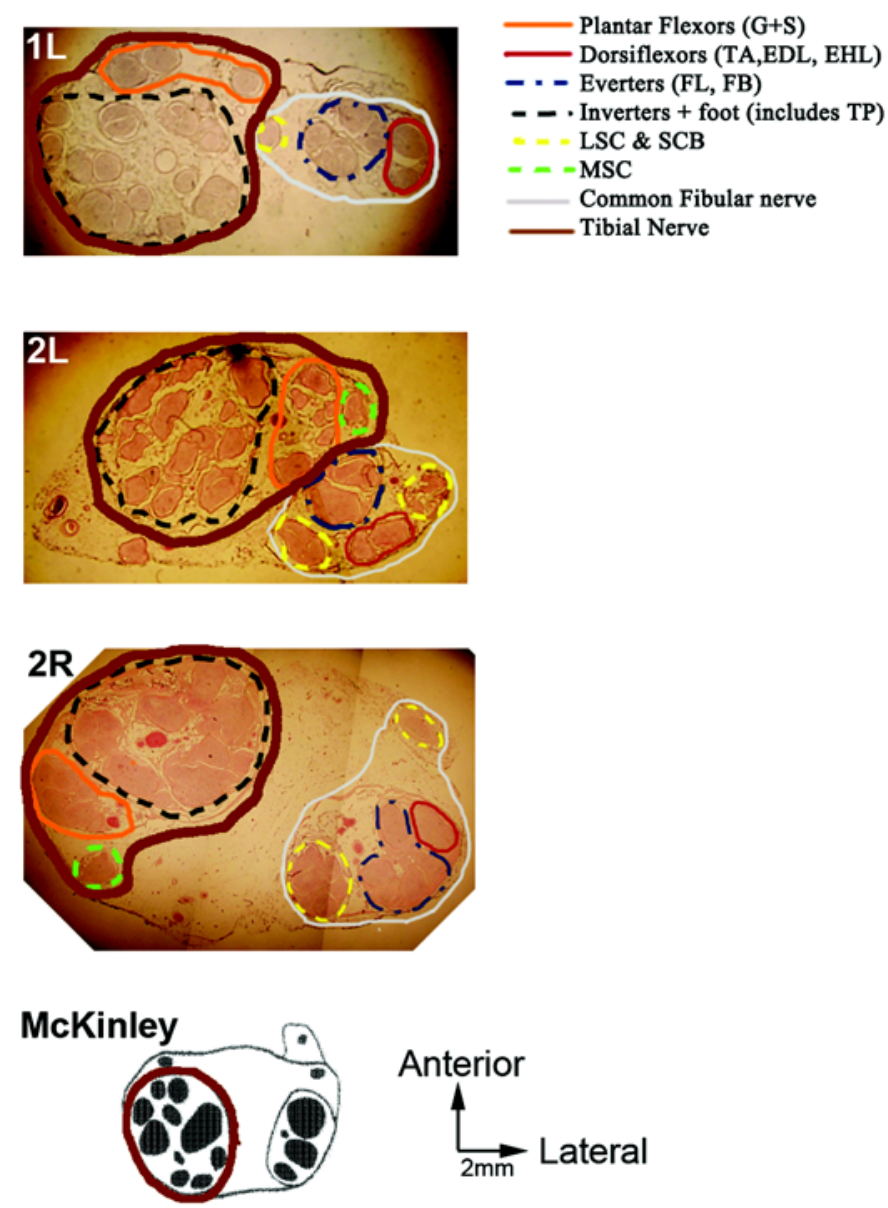

Figure 7.

Distal sciatic nerve cross-section comparison. Figure shows four lower sciatic nerve cross-sections, including one from McKinley (McKinley JC. The intraneural plexus of fasciculi and fibers in the sciatic nerve. Arch Neurol Psychiatry. 1921;6(4):377-99. Copyright (c) (1921) American Medical Association. All rights reserved.) taken proximal to sciatic bifurcation. Fascicles going to common fibular nerve are outlined in thin gray and those going to tibial nerve in thick brown lines. Thick orange outline includes fascicles innervating gastrocnemius and soleus muscles that are critical for plantar flexion of subtarsal joint. Thick red outline delineates fascicles composing deep fibular nerve, responsible for musculature producing dorsiflexion (tibialis anterior, EDL, and EHL). Yellow and green dotted outline encircles fascicles going to lateral and medial sural cutaneous (MSC) nerves, respectively. Nerve sections are oriented with lateral side to the right and anterior side to top. EDL/EHL = extensor digitorum/hallucis longus, FL/FB = fibularis longus/brevis, $G=$ gastrocnemius, $L S C=$ lateral sural cutaneous, $\mathrm{S}=$ soleus, $\mathrm{SCB}=$ sural communicating branch, $\mathrm{TA}=$ tibialis anterior, $\mathrm{TP}=$ tibialis posterior. 
Such selectivity would allow for balanced dorsiflexion through stimulation of the DF with simultaneous innervations of the SF nerve to generate eversion off-setting any inversion produced by DF stimulation. O'Halloran et al. made similar conclusions about generating pure dorsiflexion without inversion or eversion through balanced activation of two channels to the groups of fascicles he observed in the common fibular nerve [9]. Multicontact nerve-cuff electrodes implanted in humans are available and provide balanced dorsiflexion in foot-drop applications [7,11-13], supporting the observed common fibular fascicular anatomy.

In the tibial nerve, the fascicular group for plantar flexors, the remainder of the motor tibial nerve, and the MSC nerve could be distinguished separately (Figure 5). This selectivity would allow for isolated plantar flexion, or alternatively, balanced inversion via stimulation of the fascicular group containing the tibialis posterior among weak plantar flexors such as the flexor digitorum longus and flexor hallucis longus. These current findings offer means for enhancing nerve-cuff electrode functionality by adding selective plantar flexion and active push-off via tibial nerve stimulation. The addition of active plantar flexion would add a valuable level of control to improve both standing stability and active push-off for mobility.

The fascicular organization of the distal sciatic nerve suggests that ankle control may be possible at this location. In the distal sciatic nerve, fascicular groups for dorsiflexion, plantar flexion, inversion, and eversion were maintained over distances greater than $2.0 \mathrm{~cm}$, supporting nerve-cuff placement (Figure 7). While the gastrocnemius and soleus were not discernable individually at the distal sciatic (Figure 7) and proximal tibial nerve (Figure 6), electrophysiologic testing could be used to delineate their exact locations. The fascicular organization of the distal sciatic nerve is conducive to ankle control with multicontact nerve-cuff electrodes.

The three distal sciatic fascicular maps reported here are being used for computer models developing nervecuff electrodes and evaluating potential interfacing locations. Designing models of nerve-cuff electrodes to selectively stimulate distal musculature using fascicular anatomy [16] has been accomplished using similar data for the femoral nerve [22]. These studies have led to intraoperative verification of the designs and approval for human feasibility trials [24].

\section{Limitations}

Studying additional specimens from a more varied population would strenthen the generizability of the results. Although relatively few specimens were examined, comparable data are available from only two authoritative studies in the literature involving only a single sciatic nerve each [17-18]. General guidelines about peripheral nerve organization have been derived from other published accounts using small numbers of samples in the past, and the morphology and fascicular anatomy results inferred from our study can be valuable in spite of the limited number of specimens examined. The histological sectioning approach used is labor and resource intensive, which limits the number of obtainable samples. As nondestructive imaging techniques improve, faster fascicular mapping may allow in vivo evaluation.

\section{CONCLUSIONS}

This study expands the knowledge of human sciatic nerve morphology and fascicular anatomy. The fascicular separation and organization confirms the feasibility of selectively achieving dorsiflexion at both the level of the proximal common fibular nerve and the distal sciatic nerve, while also balancing inversion and eversion. The ability to provide active push-off and balance with a single implant would improve current neural prostheses to restore standing and walking. The fascicular organization suggests that selective activation of the fascicles responsible for plantar flexion can be isolated and selectively stimulated at the level of the proximal tibial nerve and the distal sciatic nerve. These morphology and fascicular anatomy results can be used to design nerve-cuff electrodes for restoration of ankle function.

\section{ACKNOWLEDGMENTS}

\section{Author Contributions:}

Study concept and design: R. J. Triolo, K. J. Gustafson. Acquisition of data: Y. Grinberg.

Analysis and interpretation of data: K. J. Gustafson, Y. Grinberg, S. Joseph, R. J. Triolo.

Drafting of manuscript: K. J. Gustafson, Y. Grinberg, S. Joseph, R. J. Triolo.

Critical revision of manuscript for important intellectual content: K. J. Gustafson, Y. Grinberg, S. Joseph, R. J. Triolo.

Statistical analysis: S. Joseph.

Obtained funding: R. J. Triolo. 
Administrative, technical, or material support: K. J. Gustafson, Y. Grinberg, S. Joseph, R. J. Triolo.

Study supervision: K. J. Gustafson, R. J. Triolo.

Financial Disclosures: The authors have declared that no competing interests exist.

Funding/Support: This material was based on work supported by grants DK077089, VA B6685R, NIH EB001889 and the Cleveland Advanced Platform Technology Center.

Additional Contributions: Special thanks to Jennifer Neville, Dustin Tyler, Brendan Masini, Matthew Stone, Yu-Tung Wong, and Matthew Schiefer for their assistance. Ms. Grinberg is now an employee at Medtronic.

\section{REFERENCES}

1. Waters RL, McNeal D, Perry J. Experimental correction of footdrop by electrical stimulation of the peroneal nerve. J Bone Joint Surg Am. 1975;57(8):1047-54. PMID:1081538

2. Strojnik P, Acimovic R, Vavken E, Simic V, Stanic U. Treatment of drop foot using an implantable peroneal underknee stimulator. Scand J Rehabil Med. 1987;19(1): 37-43. PMID:3495033

3. Kljaji M, Malezic M, Aimovi R, Vavken E, Stanic U, Pangrsic B, Rozman J. Gait evaluation in hemiparetic patients using subcutaneous peroneal electrical stimulation. Scand J Rehabil Med. 1992;24(3):121-26. PMID:1411357

4. Hansen M, Haugland M, Sinkjaer T, Donaldson N. Real time foot drop correction using machine learning and natural sensors. Neuromodulation. 2002;5(1):41-53. PMID:22151781 http://dx.doi.org/10.1046/j.1525-1403.2002._2008.x

5. Weber DJ, Stein RB, Chan KM, Loeb G, Richmond F, Rolf $\mathrm{R}$, James K, Chong SL. BIONic WalkAide for correcting foot drop. IEEE Trans Neural Syst Rehabil Eng. 2005; 13(2):242-46. PMID:16003906 http://dx.doi.org/10.1109/TNSRE.2005.847385

6. Haugland MK, Sinkjaer T. Cutaneous whole nerve recordings used for correction of footdrop in hemiplegic man. IEEE Trans Rehabil Eng. 1995;3(4):307-17. http://dx.doi.org/10.1109/86.481970

7. Kenney L, Bultstra G, Buschman R, Taylor P, Mann G, Hermens H, Holsheimer J, Nene A, Tenniglo M, Van der Aa H, Hobby J. An implantable two channel drop foot stimulator: Initial clinical results. Artif Organs. 2002;26(3): 267-70. PMID:11940030 http://dx.doi.org/10.1046/j.1525-1594.2002.06949.x

8. Lyons GM, Sinkjaer T, Burridge JH, Wilcox DJ. A review of portable FES-based neural orthoses for the correction of drop foot. IEEE Trans Neural Syst Rehabil Eng. 2002; 10(4):260-79. PMID:12611364 http://dx.doi.org/10.1109/TNSRE.2002.806832
9. O’Halloran T, Haugland M, Lyons GM, Sinkjaer T. Modified implanted drop foot stimulator system with graphical user interface for customised stimulation pulse-width profiles. Med Biol Eng Comput. 2003;41(6):701-9.

PMID:14686596

http://dx.doi.org/10.1007/BF02349978

10. Veltink PH, Slycke P, Hemssems J, Buschman R, Bultstra $\mathrm{G}$, Hermens $\mathrm{H}$. Three dimensional inertial sensing of foot movements for automatic tuning of a two-channel implantable drop-foot stimulator. Med Eng Phys. 2003;25(1):21-28. PMID:12485783 http://dx.doi.org/10.1016/S1350-4533(02)00041-3

11. Hoffer J, Baru M, Bedard S, Calderon E, Desmoulin G, Dhawan P. Initial results with fully implanted Neurostep FES system for foot drop. Proc 10th Ann Conf IFES; Montreal, Canada. p. 53-55.

12. Burridge J, Haugland M, Larsen B, Svaneborg N, Iversen H, Brogger CP, Pickering R, Sinkjaer T. Long-term followup of patients using the ActiGait implanted drop-foot stimulator. Proceedings of the 10th Annual Conference of the International Functional Electrical Stimulation Society; 2005 Jul; Montreal, Canada. p. 264-66.

13. Burridge JH, Haugland $M$, Larsen B, Pickering RM, Svaneborg N, Iversen HK, Christensen PB, Haase J, Brennum J, Sinkjaer T. Phase II trial to evaluate the ActiGait implanted drop-foot stimulator in established hemiplegia. J Rehabil Med. 2007;39(3):212-18. PMID:17468789 http://dx.doi.org/10.2340/16501977-0039

14. Naples GG, Mortimer JT, Yuen TG. Overview of peripheral nerve electrode design and implantation. In: Agnew WF, McCreery DB, editors. Neural prostheses: Fundamental studies. Englewood Cliffs (NJ): Prentice Hall; 1990. p. $107-45$.

15. Grill WM, Mortimer JT. Neural and connective tissue response to long-term implantation of multiple contact nerve cuff electrodes. J Biomed Mater Res. 2000;50(2): 215-26. PMID:10679687 http://dx.doi.org/10.1002/(SICI)10974636(200005)50:2<215::AID-JBM17>3.0.CO;2-A

16. Schiefer MA, Triolo RJ, Tyler DJ. A model of selective activation of the femoral nerve with a flat interface nerve electrode for a lower extremity neuroprosthesis. IEEE Trans Neural Syst Rehabil Eng. 2008;16(2):195-204. PMID:18403289

http://dx.doi.org/10.1109/TNSRE.2008.918425

17. McKinley JC. The intraneural plexus of fasciculi and fibers in the sciatic nerve. Arch Neurol Psychiatry. 1921;6(4): 377-99.

18. Sunderland S, Ray LJ. The intraneural topography of the sciatic nerve and its popliteal divisions in man. Brain. 1948;71(Pt 3):242-73. PMID:18099549 http://dx.doi.org/10.1093/brain/71.3.242 
19. Choi AQ, Cavanaugh JK, Durand DM. Selectivity of multiplecontact nerve cuff electrodes: A simulation analysis. IEEE Trans Biomed Eng. 2001;48(2):165-72.

PMID:11296872 http://dx.doi.org/10.1109/10.909637

20. Grill WM, Jr, Mortimer JT. Quantification of recruitment properties of multiple contact cuff electrodes. IEEE Trans Rehabil Eng. 1996;4(2):49-62. PMID:8798072 http://dx.doi.org/10.1109/86.506402

21. Tyler DJ, Durand DM. Functionally selective peripheral nerve stimulation with a flat interface nerve electrode. IEEE Trans Neural Syst Rehabil Eng. 2002;10(4):294-303. PMID:12611367 http://dx.doi.org/10.1109/TNSRE.2002.806840

22. Gustafson KJ, Pinault GC, Neville JJ, Syed IS, Davis JA, Jr, Jean-Claude J, Triolo RJ. Fascicular anatomy of human femoral nerve: Implications for neural prostheses using nerve cuff electrodes. J Rehabil Res Dev. 2009;46(7):973-84. PMID:20104420 http://dx.doi.org/10.1682/JRRD.2008.08.0097

23. Schiefer MA, Polasek KH, Triolo RJ, Pinault GC, Tyler DJ. Intraoperative demonstration of selective stimulation of the common human femoral nerve with a FINE. Proc 31st Ann Int Conf IEEE EBMS; 2009 Sep 3-6; Minneapolis, MN. p. 610-13.

24. Schiefer MA, Polasek KH, Triolo RJ, Pinault GC, Tyler DJ. Selective stimulation of the human femoral nerve with a flat interface nerve electrode. J Neural Eng. 2010;7(2): 26006. PMID:20208125 http://dx.doi.org/10.1088/1741-2560/7/2/026006

25. Moore KL, Dalley AF. Clinically Oriented Anatomy. 5th ed. Baltimore (MD): Lippincott Williams \& Wilkins; 2006. p. 555-724.

26. Okraszewska E, Migdalski L, Jedrzejewski KS, Bolanowski W. Sciatic nerve variations in some studies on the
Polish population and its statistical significance. Folia Morphol (Warsz). 2002;61(4):277-82. PMID:12725497

27. Bodily KD, Spinner RJ, Bishop AT. Restoration of motor function of the deep fibular (peroneal) nerve by direct nerve transfer of branches from the tibial nerve: An anatomical study. Clin Anat. 2004;17(3):201-5.

PMID:15042567

http://dx.doi.org/10.1002/ca.10189

28. Jabaley ME, Wallace WH, Heckler FR. Internal topography of major nerves of the forearm and hand: A current view. J Hand Surg Am. 1980;5(1):1-18. PMID:7365209

29. Gustafson KJ, Zelkovic PF, Feng AH, Draper CE, Bodner DR, Grill WM. Fascicular anatomy and surgical access of the human pudendal nerve. World J Urol. 2005;23(6):411-18. PMID:16333625 http://dx.doi.org/10.1007/s00345-005-0032-4

30. Waters RL, McNeal DR, Faloon W, Clifford B. Functional electrical stimulation of the peroneal nerve for hemiplegia. Long-term clinical follow-up. J Bone Joint Surg Am. 1985;67(5):792-93. PMID:3873456

Submitted for publication October 16, 2010. Accepted in revised form May 16, 2011.

This article and any supplementary material should be cited as follows:

Gustafson KJ, Grinberg Y, Joseph S, Triolo RJ. Human distal sciatic nerve fascicular anatomy: Implications for ankle control using nerve-cuff electrodes. J Rehabil Res Dev. 2012;49(2):309-22. http://dx.doi.org/10.1682/JRRD.2010.10.0201

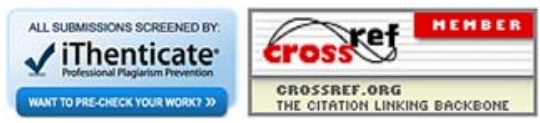


